\title{
Management of stage IIIA NSCLC: the role of radiotherapy-a narrative review
}

\author{
Thomas Iseli ${ }^{1}$, Krisztian Süveg ${ }^{1}$, Galina Farina Fischer ${ }^{1,2}$, Markus Glatzer ${ }^{1}$, Paul Martin Putora ${ }^{1,3}$ \\ ${ }^{1}$ Department of Radiation Oncology, Kantonsspital St. Gallen, St. Gallen, Switzerland; ${ }^{2}$ Department of Radiation Oncology, Kantonsspital \\ Winterthur, Winterthur, Switzerland; ${ }^{3}$ Department of Radiation Oncology, University of Bern, Bern, Switzerland \\ Contributions: (I) Conception and design: T Iseli, PM Putora; (II) Administrative support: None; (III) Provision of study materials or patients: None; \\ (IV) Collection and assembly of data: All authors; (V) Data analysis and interpretation: All authors; (VI) Manuscript writing: All authors; (VII) Final \\ approval of manuscript: All authors. \\ Correspondence to: Thomas Iseli. Department of Radiation Oncology, Kantonsspital St. Gallen, Rorschacherstrasse 95, 9007 St. Gallen, Switzerland. \\ Email: thomas.iseli@kssg.ch.
}

\begin{abstract}
Worldwide, two million patients are newly diagnosed with lung cancer and 1.7 million die of it every year. The most relevant risk factor is and remains smoking. Non-small cell lung cancer (NSCLC) is the most common histology and accounts for up to $85 \%$ of lung cancer cases. The $8^{\text {th }}$ TNM classification defines stages I to IV for NSCLC. However, stage III consists of a heterogeneous group of situations, combining different T- and N-stages into subgroups IIIA-C. Furthermore, the Robinson classification stratifies stage IIIA N2 into stage IIIA1-4. Depending on the nodal extent, patients may qualify for different treatment modalities or their combinations. Surgery, chemotherapy, radiotherapy, and immunotherapy play a key role in treatment decisions. Evidence guides lower N-stages (N0-1) to surgical treatment and unresectable patients (N3) to definitive chemoradiotherapy (CRT). Optimal treatment for N2-disease remains controversial. Bimodal treatment including chemotherapy and surgery or definitive CRT are standards of care. Neither concept could prove superiority over the other treatment approach in clinical trials, this equipoise is reflected in clinical guidelines. Most current guidelines do not recommend a single treatment strategy but emphasise the need of a multidisciplinary team for decision making as multiple options exist. A small minority of guidelines define trimodal therapy as a standard of care for selected patients. In a setting with multiple treatment options, patient-preference should play a very prominent role in decision making.
\end{abstract}

Keywords: Lung cancer; non-small cell lung cancer (NSCLC); stage IIIA; radiotherapy

Received: 20 May 2020; Accepted: 26 February 2021; Published: 25 May 2022.

doi: $10.21037 /$ ccts-20-101

View this article at: http://dx.doi.org/10.21037/ccts-20-101

\section{Introduction}

Worldwide, two million patients are newly diagnosed with lung cancer and 1.7 million die of it every year (1). The most relevant risk factor is and remains smoking $(2,3)$. Non-small cell lung cancer (NSCLC) is the most common histology and accounts for up to $85 \%$ of lung cancer cases (4). The $8^{\text {th }}$ edition of the TNM-classification defines stages I to IV, with subgroups to each stage (Table 1) (5). Generally, patients with stage I to III qualify for curative treatment regimens, while stage IV patients undergo palliative treatment-individual exemptions excluded. As symptoms occur late, $20-27 \%$ of patients are diagnosed with locally advanced disease (LANSCLC-stage III) $(6,7)$. Patients with LA-NSCLC show large variety of primary tumour extent or lymph node (LN) involvement and are all staged as IIIA-C. Especially within stage III N2 disease, the patient collective shows considerable heterogeneity, leading to different treatment options. Main multimodal treatment options are surgery with (neo)adjuvant chemotherapy or neoadjuvant chemoradiotherapy (CRT) or definitive CRT with or without immunotherapy (IT) (8). Nevertheless, overall survival (OS) remains poor for LA- 
Table 1 Staging according to the $8^{\text {th }}$ edition of the TNM-classification adapted from Goldstraw et al. (5)

\begin{tabular}{lllll}
\hline Staging & NO & N1 & N2 & N3 \\
\hline T1 & IA & IIB & IIIA & IIIB \\
T2a & IB & IIB & IIIA & IIIB \\
T2b & IIA & IIB & IIIB \\
T3 & IIB & IIIA & IIIB & IIIC \\
T4 & IIIA & IIIA & IIIB & IIIC \\
M1a & IVA & IVA & IVA \\
M1b & IVA & IVA & IVA \\
M1c & IVB & IVB & IVB \\
\hline
\end{tabular}

NSCLC. For N2 disease (IIIA/IIIB) it is in the range of $15-40 \%$ at 5 years (9-11). As the optimal treatment choice for N2 IIIA NSCLC remains controversial, this narrative review focuses on the role of radiation therapy (RT) in the multimodal treatment of LA-NSCLC, especially stage IIIA with N2 disease. We present the following article in accordance with the Narrative Review reporting checklist (available at https://ccts.amegroups.com/article/ view/10.21037/ccts-20-101/rc).

\section{Methods}

Relevant publications for this review were retrieved from PubMed using the terms "NSCLC", "stage III", "radiotherapy" and "radiation therapy". Furthermore, international guidelines by the American College of Chest Physicians (ACCP), National Comprehensive Cancer Network (NCCN), European Society for Medical Oncology (ESMO), National Institute for Health and Care Excellence (NICE) or the German S3 guidelines were consulted for statements in this review. Publications in English or German were considered.

\section{Stage III NSCLC}

The TNM classification defines different stage III diseases (IIIA-C) based on the $\mathrm{T}$ and $\mathrm{N}$ status (Table 1). Nodal staging plays a key role in subdividing stage III and determines in parts the further clinical management. While ipsilateral peri-bronchial and/or hilar LNs are classified as N1 disease, involvement of ipsilateral mediastinal and/or subcarinal LNs is classified as N2. Metastases in contralateral mediastinal or hilar LNs as well as any scalene/supraclavicular LN involvement are classified as N3. Stage IIIA is defined as T4N0, T3-4N1 or includes mediastinal LN involvement for T1-2N2. With N2 disease, T3-4 tumours are staged IIIB, representing an ipsilaterally confined disease. Stage IIIB also includes N3 disease with lower $\mathrm{T}$ stages. The most unfavourable constellations without distant metastases, T3-4 N3, are grouped in stage IIIC. Five-year survival rates for IIIA are 36\%, for IIIB $26 \%$ and for IIIC $13 \%$ (5).

Within stage IIIA, the extent of nodal involvement can differ significantly. This may influence the treatment choice, as this stage is considered potentially resectable. Stage IIIA can be further classified into four subsets according to the Robinson classification (12). While in stage IIIA1 and IIIA2, intraoperatively micro- or macrometastases are found respectively, IIIA3 has (preoperatively) known single- or multi-station involvement. IIIA4 presents with fixed multistation bulky N2 disease. One further subgroup within stage IIIA should be mentioned. Due to the proximity to adjacent structures such as the brachial plexus or vessels, tumours of the superior sulcus (termed Pancoast tumours when they present with corresponding symptoms-Horner's triad) are often managed with a site specific approach (13-15).

\section{Treatment for stage III NSCLC}

\section{Basics of RT}

In order to prepare for radiotherapy, a CT scan in treatment position is performed (planning CT). Different auxiliaries can be used to create reproducible positioning. Cameras can track patients' chest wall movements while breathing, allowing to track tumour movement through several scans (4D-CT). Depending on tumour-movement, physicians 

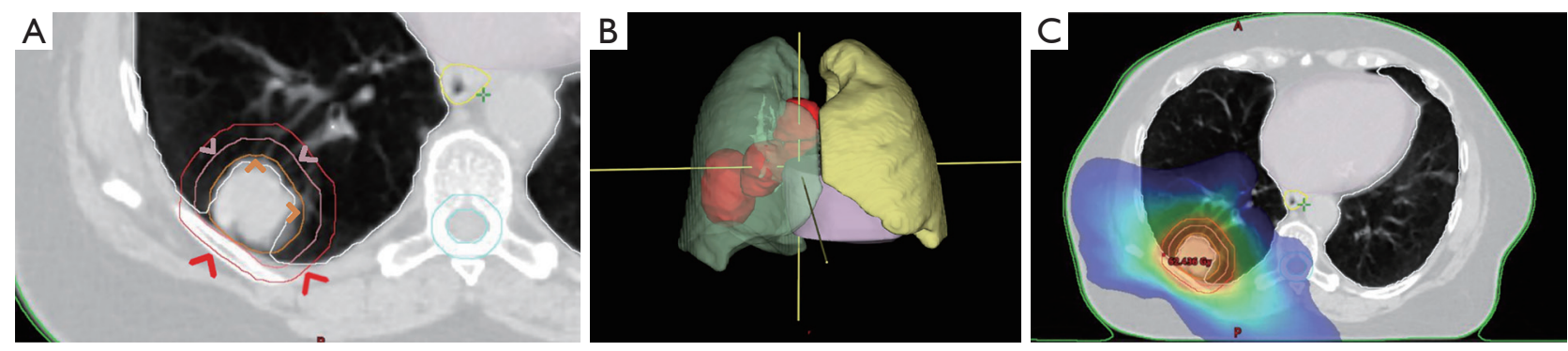

Figure 1 Illustration of planning steps in modern RT. (A) Delineation of GTV (orange arrows), CTV (pink arrows) and PTV (red arrows). (B) 3D model of the PTV (red), lungs in green/yellow. (C) Colourwash of dose distribution, ranging from 20 Gy (blue) to 62.4 (red). RT, radiation therapy; GTV, gross tumour volume; CTV, clinical target volume; PTV, planning target volume.

may choose free breathing or certain phases of the breathing cycle ("gating") for treatment delivery. Some patients can be treated in "breath hold", where treatment is applied only while patients hold their breath after forced inspiration or exspiration. If fiducial markers are inserted into the tumour, movement can be tracked in real-time allowing optimisation of radiation delivery accordingly. These techniques minimise the overall volume treated and help to reduce irradiation of the healthy lungs. Imaging, desirably a PET/ CT scan and clinical information are used to delineate the tumour on the planning CT scan [= gross tumour volume (GTV)] (16). The GTV is enlarged for microscopic spread [= clinical target volume (CTV)] and positioning inaccuracy [= planning target volume (PTV)] (16). Treatment is usually delivered using linear accelerators. Through rotation of the gantry and positioning of small metal leaves (multileaf collimator) the radiation beam is formed and allows precise delivery to the treated volume. This technique is known as intensity modulated radiotherapy (IMRT) or volume modulated arc therapy (VMAT) and often achieves better sparing of organs at risk (Figure 1) than older techniques (3D conformal radiotherapy). Before treatment delivery, a CT or $\mathrm{kV}$-radiograph in treatment position can be performed to ensure correct positioning. Due to the typically large treatment fields and significant volumes of healthy tissues within the treatment fields, stereotactic body radiotherapy (SBRT) is not applied in stage III lung cancer.

\section{Current treatment for stage III}

In general, data from studies on stage III NSCLC need to be interpreted cautiously. Different confounders such as heterogeneous staging methods, interpretation of nodal extent (bulky $v s$. non-bulky) or under-representation of subgroups may distort results. Several studies, justifying todays established treatment rationales, were conducted in the pre-PET/CT era. Today's preoperative diagnostics, including endosonographic biopsies (e.g., through endobronchial ultrasound guidance), allow for a potentially better assessment of nodal extension. Among the most important decision-making parameters are LN spread, patients' morbidity and patient preference.

If surgical management is considered, complete resection with negative margins is a top priority. In case of positive margins, postoperative RT (PORT) appears reasonable, evidence is still limited though. This option should be carefully discussed in a multidisciplinary team to potentially decrease the risk of local recurrence after individual risk assessment (17). For patients deemed unresectable, definitive CRT is advised (18). Platinumbased chemotherapies are an established standard of care $(17,19)$. Sequential CRT showed improved OS over RT alone (20) and the comparison of sequential to concomitant CRT favoured the latter $(21,22)$.

The most recent relevant trial defining the current standard of care was the RTOG 0617 trial (23). This two-by-two study compared standard dose CRT (60 Gy) with dose escalated CRT (74 Gy) with or without cetuximab-a chimeric antibody targeted at the EGFR receptor. Five hundred and forty-four patients with stage IIIA and IIIB were included. The addition of cetuximab did not improve outcomes. Interestingly, standard dose CRT (60 Gy) performed better, with a median OS of $28.7 v s .20 .3$ months (HR: 1.38, $\mathrm{P}=0.004)$. No significant differences in failure pattern neither local nor distant, were found between the two dose-levels. For patients unsuitable for CRT, individual approaches, including sequential radiotherapy, radiotherapy alone or systemic therapy alone (including 
IT) can be considered $(18,24)$. While N0-1 is typically approached with surgery and N3 with radiotherapy, N2 disease potentially allows for both in a multimodal treatment and needs to be assessed carefully (8).

\section{N2 disease}

The collective of patients staged as IIIA and IIIB disease, represents a broad range of clinical scenarios. Independent of LN extent, tumour location and size can impact on morbidity of treatments and influence resectability. Stage III N2 is often referred to as potentially resectable, as patients need to be evaluated individually, if deemed resectable and operable. Thanks to technical advances and changes in operative techniques [e.g., video assisted thoracic surgery (VATS)], perioperative outcomes improved significantly $(25,26)$.

The EORTC 08941 trial (9) included pathologically proven N2 NSCLC patients deemed unresectable at presentation. Guidelines for unresectability were as follows: (I) any N2 involvement by a nonsquamous cell carcinoma; (II) in case of squamous cell carcinoma, any N2 nodal involvement exceeding level $4 \mathrm{R}$ for a right-sided tumour and level 5 and 6 for a left-sided tumour. Patients with at least minimal radiological treatment response after a minimum of two cycles of induction chemotherapy, were randomised to either surgery or RT (60-62.5 Gy). Sixtyone percent of the 579 patients showed a response and were allocated to either arm. Median OS at 5 years was $15.7 \%$ for the surgical and $14 \%$ for the radiation arm. In comparison, the radiation arm showed more locoregional failures compared to surgery. In $49.7 \%$ of resected patients, no residual tumour was seen (R0 resection) (27). Forty percent of operated patients with residual tumour (R1/2 resection) received PORT (56 Gy). However, there was no OS benefit compared to patients with residual tumour without PORT.

Patients with mediastinoscopy proven IIIA N2 disease deemed primarily resectable were randomised in the intergroup 0139 trial to CRT induction therapy (45 Gy) followed by either surgery or radiotherapy up to $61 \mathrm{~Gy}$ (10). The study failed to demonstrate significant survival benefits for either arm (27\% for surgery $v s .20 \%$ for CRT at 5 years, $\mathrm{P}=0.10$ ), but showed better progression-free survival (PFS) for patients in the surgical arm at 2 years $(22 \% v s$. $11 \%, \mathrm{P}=0.017)$. A secondary analysis led to the hypothesis that patients may benefit from trimodal treatment, if the surgical procedure performed is lobectomy instead of pneumonectomy. This was in light of the mortality observed with pneumonectomies $v s$. lobectomies (22\% for simple and
$29 \%$ for complex pneumonectomies compared to $1 \%$ for lobectomies), the highest risk being associated with rightsided pneumonectomies. Ten out of the 16 deaths in the surgical arm, occurred within 30 days after surgery (28). It may be argued that a selection of patients especially suitable for radiotherapy would show a similar improvement. In the meantime, high-volume centres published better results for post-induction pneumonectomy, although these findings were heterogeneous $(29,30)$. The equipoise of these results (Intergroup 0139 and EORTC 08941) was shown in two meta-analyses including other trials $(31,32)$. While a higher treatment associated mortality was observed in surgical studies (risk ratio $=3.56, \mathrm{P}=0.0005$ ) (32), both analysis concluded definitive CRT and bimodal treatment including surgery to be comparable local treatment options for patients with $\mathrm{N} 2$ disease.

The ESPATUE trial restaged patients with resectable IIIA and selected IIIB NSCLC (N3 disease with contralateral mediastinal nodes or proven $\mathrm{T} 4$ disease with involvement of the pulmonary artery, carina, left atrium, vena cava or mediastinum-according the $6^{\text {th }}$ edition of the TNM) after concomitant CRT with 1.5 Gy twice daily to 45 Gy. Resectable patients were randomised to surgery or CRT Boost (65 to $71 \mathrm{~Gy}$, risk adapted) (11). In the surgical arm, pneumonectomies were performed in $32.8 \%$ of the cases. Overall, R0 resections were achieved in $81 \%$ after induction CRT. OS showed no difference at 5 years with $44 \%$ for surgery and $40 \%$ for CRT. Neither did PFS differ significantly with $32 \%$ vs. $35 \%$, respectively. No unexpected toxicity was shown. Especially in the surgical arm, postoperative deaths were lower than previously published, as e.g., in the intergroup 0139 trial (4 after lobectomy, 1 after bi-lobectomy and none after pneumonectomy). CRT showed acceptable toxicity with grade 3 and 4 leukopenia comparable between the arms. Esophagitis in the CRT arm was seen as grade 3 in $26 \%$ of patients, but no grade 4 was reported.

\section{Postoperative radiotherapy}

PORT may play a role in the management of stage III NSCLC. Although an older meta-analysis (33) showed detrimental effects on OS for stage I and II, patients with stage III or N2 nodal involvement showed no disadvantage (33). Overall risk of local recurrence was reduced by $24 \%$ with PORT - the benefit however, may be smaller due to competing risk and higher mortality. In pN2 disease, PORT remained controversial. In 2008, a retrospective analysis of the ANITA trial (34) was performed, where RT was 
recommended in patients with pathologically positive nodes (45-60 Gy, 2 Gy per fraction) (35). There was a benefit in 5-year OS, in pN2 disease with radiotherapy. Based on this data, a selected collective of $\mathrm{N} 2$ positive patients was considered to benefit from PORT after complete resection. To answer this question, the Lung ART trial (ClinicalTrials. gov Identifier: NCT00410683), a prospective randomised trial was initiated. Recently, first data was published at ESMO 2020. No significant difference in disease free survival (DFS) was seen between PORT and the control arm with $47.1 \%$ and $43.8 \%$, respectively. For IIIA N2, a non-significant benefit in DFS of $15 \%$ was seen. OS was comparable at 3 years, $66.5 \%$ with PORT vs. $68.5 \%$ without it. The present data rules out PORT after complete resection as standard of care for all stage III patients, further analysis is however needed to possibly identify subgroups potentially benefiting of PORT.

Several publications try to rationalise the optimal treatment for IIIA N2 disease based on available randomised clinical trials. In 2019, Zhao et al. published a network meta-analysis comparing all combinations of chemotherapy, RT and surgery (36). A total of 18 trials were included in the analysis and identified treatments showing best OS outcome with least treatment related deaths. The authors found neoadjuvant chemotherapy followed by surgery and adjuvant RT as well as neoadjuvant chemotherapy followed by surgery and adjuvant chemotherapy to be the most favourable ones. This analysis underlines the importance of chemotherapy in this setting. The results need to be interpreted cautiously, as heterogeneity among the patients in the studies, small numbers of participants and long time span with substantial advances in surgery and RT may have distorted the results. While such analyses may provide certain insight, they do not supersede the results of randomised controlled trials.

\section{IT and synergies with $R T$}

NSCLC and the patients' immune system have significant interactions. The tumour is capable of establishing an immunosuppressive microenvironment and releasing immunosuppressive cytokines (37-40). These mechanisms of evasion may be affected by irradiation, due to immunemediation, both at the treatment site and potentially distant sites (so called "abscopal effect"). This effect is possibly explained by up-regulation of several cellular death receptors and induced immunogenic cell death (41). RT may induce increased expression of PD-L1, which might explain the good results achieved by the PACIFIC trial (18). This phase III study for unresectable stage III NSCLC showed a benefit in OS for adjuvant IT with Durvalumab after definitive CRT (18). Seven hundred and thirteen patients were randomised 2:1 to receive Durvalumab or a placebo control. After 2 years, OS was $66.3 \%$ for the Durvalumab arm and $55.6 \%$ in the placebo group. Furthermore, PFS was 17.2 vs. 5.6 months in favour of Durvalumab. It was associated with slightly higher grade 3 and 4 adverse effects, but overall, toxicity was not increased significantly.

\section{Current guidelines}

Multiple guidelines exist and show consensus on the treatment for LA-NSCLC. For example, the newer consensus of Asian societies, shows high concordance with all recommendations of the 2017 ESMO guidelines accepting them as clinical practice guidelines (42). Most guidelines agree that patients with LA-NSCLC need to be discussed in multidisciplinary boards attended by an experienced thoracic surgeon and radiation oncologist and the best treatment remains a matter of debate (8). Main criteria for initial decision-making include expected $\mathrm{R} 0$ resection. If the tumour is considered unresectable or a patient non-operable, guidelines agree on CRT as the standard of care $(17,43)$. In the other cases with N2disease, guidelines typically do not prefer one treatment over another (8). It should be mentioned that several recent guidelines recommend considering trimodal treatment as an option and even recommend trimodal treatment as a first choice for patients, fit enough to tolerate neoadjuvant CRT $(44,45)$. Especially the frequently used distinction of single $v s$. multistation $\mathrm{N} 2$ extent, needs to be considered cautiously. It is controversial as the results from the EORTC 08941 (high-volume largely multistation N2) and the Intergroup 0139 (76\% of participants with single station N2) showed no difference in OS $(9,10,46)$. Among most guidelines, "bulky" vs. "non-bulky" had influenced treatment recommendations significantly. Consensus shifts toward RT in bulky LNs (8), yet a universally accepted definition of "bulky" is missing.

For resectable, or potentially resectable superior sulcus tumours, guidelines recommend induction CRT followed by resection in case of response to induction treatment. This recommendation is largely based on results of the Intergroup 0160 trial, a single-arm phase II study including 110 patients with T3-4N0-1 superior sulcus tumours (14). 
Patients with a complete pathological remission had a significant OS benefit.

In current guidelines PORT is considered potentially beneficial for selected patients with proven $\mathrm{pN} 2$ disease. As these benefits arise from an ad-hoc analysis no guideline recommends PORT routinely. Newer data from the Lung ART trial will probably lead to recommendations against PORT in completely resected patients.

\section{Limitations and other factors}

The evidence for various treatment options in stage IIIA NSCLC has several limitations. Crucial trials were conducted before PET/CTs or endobronchial ultrasound were routinely used in staging. Furthermore, in some trials, such as the ESPATUE trial, up to a third of the included patients had T4 N0/1 NSCLC. Such factors make it difficult to draw conclusions for a more specific subgroup such as TNM $8^{\text {th }}$ IIIA.

With different options available, choosing the best treatment for each individual patient remains difficult. Besides medical criteria, the patients' preference shifts into attention (47). It is essential to consider individual preferences while actively engaging in a shared decisionmaking process with the patient. While side effects are relevant for the decision-making of patients, unfortunately side-effect reporting has been rather poor in historical trials (48). Hopefully side effects and quality of life will be better recorded and reported in future trials.

The authors of this review were selected to provide the view of radiation oncologists, which in itself exposes this manuscript to the risk of specialty bias.

\section{Summary}

The presented data shows different treatment options for stage IIIA N2. Choice of treatment remains a matter of debate. Multimodal therapies including surgery and definitive CRT have been shown to be similarly effective. Therefore, patients with stage IIIA disease can be treated with CRT or a surgical approach (bi- or trimodal). Treatment should be discussed upfront in a multidisciplinary team. Potentially resectable superior sulcus tumours typically receive induction CRT followed by resection in case of treatment response. Patients with N3 should be offered CRT. In case of N2 disease further differentiation is needed. For unforeseen intraoperative N2 disease (IIIA1-2) adjuvant chemotherapy is indicated. Primarily unresectable
N2 disease (IIIA4) qualifies for definitive CRT. For patients, presenting with potentially resectable N2 disease (IIIA3), definitive CRT and surgery are both good options and need to be assessed individually by a multidisciplinary team and with the patient preference in mind. PORT cannot be recommended routinely for completely resected $\mathrm{N} 2$ disease. In case of incomplete resection, decisions should be made individually in a multidisciplinary tumour board. IT, in combination with RT, shows promising synergistic effects and has been shown to improve OS in the adjuvant setting.

\section{Acknowledgments}

Funding: None.

\section{Footnote}

Provenance and Peer Review: This article was commissioned by the Guest Editors (Fabrizio Minervini and Marco Scarci) for the series "Controversies in the Management of Stage IIIA Non-Small-Cell Lung Cancer" published in Current Challenges in Thoracic Surgery. The article has undergone external peer review.

Reporting Checklist: The authors have completed the Narrative Review reporting checklist. Available at https:// ccts.amegroups.com/article/view/10.21037/ccts-20-101/rc

Peer Review File: Available at https://ccts.amegroups.com/ article/view/10.21037/ccts-20-101/prf

Conflicts of Interest: All authors have completed the ICMJE uniform disclosure form (available at https://ccts. amegroups.com/article/view/10.21037/ccts-20-101/coif). The series "Controversies in the Management of Stage IIIA Non-Small-Cell Lung Cancer" was commissioned by the editorial office without any funding or sponsorship. PMP reports grants from AstraZeneca, Celgene and Takeda to the department, outside the submitted work. The authors have no other conflicts of interest to declare.

Ethical Statement: The authors are accountable for all aspects of the work in ensuring that questions related to the accuracy or integrity of any part of the work are appropriately investigated and resolved.

Open Access Statement: This is an Open Access article distributed in accordance with the Creative Commons 
Attribution-NonCommercial-NoDerivs 4.0 International License (CC BY-NC-ND 4.0), which permits the noncommercial replication and distribution of the article with the strict proviso that no changes or edits are made and the original work is properly cited (including links to both the formal publication through the relevant DOI and the license). See: https://creativecommons.org/licenses/by-nc-nd/4.0/.

\section{References}

1. Cancer. World Health Organization (WHO). 2020. Available online: https://www.who.int/news-room/factsheets/detail/cancer (Accessed April 04, 2020).

2. Malhotra J, Malvezzi M, Negri E, et al. Risk factors for lung cancer worldwide. Eur Respir J 2016;48:889-902.

3. Molina JR, Yang P, Cassivi SD, et al. Non-small cell lung cancer: epidemiology, risk factors, treatment, and survivorship. Mayo Clin Proc 2008;83:584-94.

4. Duma N, Santana-Davila R, Molina JR. Non-small cell lung cancer: epidemiology, screening, diagnosis, and treatment. Mayo Clin Proc 2019;94:1623-40.

5. Goldstraw P, Chansky K, Crowley J, et al. The IASLC lung cancer staging project: proposals for revision of the TNM stage groupings in the forthcoming (eighth) edition of the TNM classification for lung cancer. J Thorac Oncol 2016;11:39-51.

6. Stage distribution in lung cancer. Canadian Partnership against cancer. 2020. Available online: https://www. systemperformance.ca/disease-sites/lung/stagedistribution/ (Accessed April 04, 2020).

7. Morgensztern D, Ng SH, Gao F, et al. Trends in stage distribution for patients with non-small cell lung cancer: a national cancer database survey. J Thorac Oncol 2010;5:29-33.

8. Putora PM, Leskow P, McDonald F, et al. International guidelines on stage III N2 nonsmall cell lung cancer: surgery or radiotherapy? ERJ Open Res 2020;6:00159-2019.

9. van Meerbeeck JP, Kramer GW, Van Schil PE, et al. Randomized controlled trial of resection versus radiotherapy after induction chemotherapy in stage IIIA-N2 non-small-cell lung cancer. J Natl Cancer Inst 2007;99:442-50.

10. Albain KS, Swann RS, Rusch VW, et al. Radiotherapy plus chemotherapy with or without surgical resection for stage III non-small-cell lung cancer: a phase III randomised controlled trial. Lancet 2009;374:379-86.

11. Eberhardt WE, Pöttgen C, Gauler TC, et al. Phase
III study of surgery versus definitive concurrent chemoradiotherapy boost in patients with resectable stage IIIA(N2) and selected IIIB non-small-cell lung cancer after induction chemotherapy and concurrent chemoradiotherapy (ESPATUE). J Clin Oncol 2015;33:4194-201.

12. Robinson LA, Ruckdeschel JC, Wagner H Jr, et al. Treatment of non-small cell lung cancer-stage IIIA: ACCP evidence-based clinical practice guidelines. Chest 2007;132:243S-65S.

13. Buderi SI, Shackcloth M, Woolley S. Does induction chemoradiotherapy increase survival in patients with Pancoast tumour? Interact Cardiovasc Thorac Surg 2016;23:821-5.

14. Rusch VW, Giroux DJ, Kraut MJ, et al. Induction chemoradiation and surgical resection for superior sulcus non-small-cell lung carcinomas: long-term results of Southwest Oncology Group Trial 9416 (Intergroup Trial 0160). J Clin Oncol 2007;25:313-8.

15. Komaki R, Erasmus J, Fujimoto J, et al. Definition and management of superior sulcus tumors. Ann Thorac Oncol Res 2018;1:1012.

16. Nestle U, De Ruysscher D, Ricardi U, et al. ESTRO ACROP guidelines for target volume definition in the treatment of locally advanced non-small cell lung cancer. Radiother Oncol 2018;127:1-5.

17. Postmus PE, Kerr KM, Oudkerk M, et al. Early and locally advanced non-small-cell lung cancer (NSCLC): ESMO Clinical Practice Guidelines for diagnosis, treatment and follow-up. Ann Oncol 2017;28:iv1-21.

18. Antonia SJ, Villegas A, Daniel D, et al. Overall survival with durvalumab after chemoradiotherapy in stage III NSCLC. N Engl J Med 2018;379:2342-50.

19. National Comprehensive Cancer Network. Non-small cell lung cancer (version 3.2020). 2020. Available online: https://www.nccn.org/professionals/physician_gls/pdf/ nscl.pdf (Accessed April 04, 2020).

20. Dillman RO, Seagren SL, Propert KJ, et al. A randomized trial of induction chemotherapy plus high-dose radiation versus radiation alone in stage III non-small-cell lung cancer. N Engl J Med 1990;323:940-5.

21. Curran WJ Jr, Paulus R, Langer CJ, et al. Sequential vs. concurrent chemoradiation for stage III non-small cell lung cancer: randomized phase III trial RTOG 9410. J Natl Cancer Inst 2011;103:1452-60. Erratum in: J Natl Cancer Inst 2012;104:79.

22. Hanna N, Neubauer M, Yiannoutsos C, et al. Phase III study of cisplatin, etoposide, and concurrent chest 
radiation with or without consolidation docetaxel in patients with inoperable stage III non-small-cell lung cancer: the Hoosier Oncology Group and U.S. Oncology. J Clin Oncol 2008;26:5755-60.

23. Bradley JD, Paulus R, Komaki R, et al. Standard-dose versus high-dose conformal radiotherapy with concurrent and consolidation carboplatin plus paclitaxel with or without cetuximab for patients with stage IIIA or IIIB non-smallcell lung cancer (RTOG 0617): a randomised, two-by-two factorial phase 3 study. Lancet Oncol 2015;16:187-99.

24. Reck M, Rodríguez-Abreu D, Robinson AG, et al. Pembrolizumab versus chemotherapy for PD-L1-positive non-small-cell lung cancer. N Engl J Med 2016;375:1823-33.

25. Falcoz PE, Puyraveau M, Thomas PA, et al. Video-assisted thoracoscopic surgery versus open lobectomy for primary non-small-cell lung cancer: a propensity-matched analysis of outcome from the European Society of Thoracic Surgeon database. Eur J Cardiothorac Surg 2016;49:602-9.

26. Jiang G, Yang F, Li X, et al. Video-assisted thoracoscopic surgery is more favorable than thoracotomy for administration of adjuvant chemotherapy after lobectomy for non-small cell lung cancer. World J Surg Oncol 2011;9:170.

27. Van Schil P, Van Meerbeeck J, Kramer G, et al. Morbidity and mortality in the surgery arm of EORTC 08941 trial. Eur Respir J 2005;26:192-7.

28. Albain KS, Swann RS, Rusch VR, et al. Phase III study of concurrent chemotherapy and radiotherapy (CT/RT) vs CT/RT followed by surgical resection for stage IIIA(pN2) non-small cell lung cancer (NSCLC): Outcomes update of North American Intergroup 0139 (RTOG 9309). J Clin Oncol 2005;23:abstr 7014.

29. Casiraghi M, Guarize J, Sandri A, et al. Pneumonectomy in stage IIIA-N2 NSCLC: should it be considered after neoadjuvant chemotherapy? Clin Lung Cancer 2019;20:97-106.e1.

30. d'Amato TA, Ashrafi AS, Schuchert MJ, et al. Risk of pneumonectomy after induction therapy for locally advanced non-small cell lung cancer. Ann Thorac Surg 2009;88:1079-85.

31. McElnay PJ, Choong A, Jordan E, et al. Outcome of surgery versus radiotherapy after induction treatment in patients with N2 disease: systematic review and metaanalysis of randomised trials. Thorax 2015;70:764-8.

32. Pöttgen C, Eberhardt W, Stamatis G, et al. Definitive radiochemotherapy versus surgery within multimodality treatment in stage III non-small cell lung cancer (NSCLC) - a cumulative meta-analysis of the randomized evidence.
Oncotarget 2017;8:41670-8.

33. Postoperative radiotherapy in non-small-cell lung cancer: systematic review and meta-analysis of individual patient data from nine randomised controlled trials. PORT Metaanalysis Trialists Group. Lancet 1998;352:257-63.

34. Douillard JY, Rosell R, De Lena M, et al. Adjuvant vinorelbine plus cisplatin versus observation in patients with completely resected stage IB-IIIA non-small-cell lung cancer (Adjuvant Navelbine International Trialist Association [ANITA]): a randomised controlled trial. Lancet Oncol 2006;7:719-27.

35. Douillard JY, Rosell R, De Lena M, et al. Impact of postoperative radiation therapy on survival in patients with complete resection and stage I, II, or IIIA non-smallcell lung cancer treated with adjuvant chemotherapy: the adjuvant Navelbine International Trialist Association (ANITA) Randomized Trial. Int J Radiat Oncol Biol Phys 2008;72:695-701.

36. Zhao Y, Wang W, Liang H, et al. The optimal treatment for stage IIIA-N2 non-small cell lung cancer: a network meta-analysis. Ann Thorac Surg 2019;107:1866-75.

37. Brahmer JR. Harnessing the immune system for the treatment of non-small-cell lung cancer. J Clin Oncol 2013;31:1021-8.

38. Tini P, Nardone V, Pastina $\mathrm{P}$, et al. The effects of radiotherapy on the survival of patients with unresectable non-small cell lung cancer. Expert Rev Anticancer Ther 2018;18:593-602.

39. Korkolopoulou P, Kaklamanis L, Pezzella F, et al. Loss of antigen-presenting molecules (MHC class I and TAP-1) in lung cancer. Br J Cancer 1996;73:148-53.

40. Domagala-Kulawik J, Osinska I, Hoser G. Mechanisms of immune response regulation in lung cancer. Transl Lung Cancer Res 2014;3:15-22.

41. Levy A, Chargari C, Marabelle A, et al. Can immunostimulatory agents enhance the abscopal effect of radiotherapy? Eur J Cancer 2016;62:36-45.

42. Park K, Vansteenkiste J, Lee KH, et al. Pan-Asian adapted ESMO Clinical Practice Guidelines for the management of patients with locally-advanced unresectable non-smallcell lung cancer: a KSMO-ESMO initiative endorsed by CSCO, ISMPO, JSMO, MOS, SSO and TOS. Ann Oncol 2020;31:191-201.

43. Ramnath N, Dilling TJ, Harris LJ, et al. Treatment of stage III non-small cell lung cancer: diagnosis and management of lung cancer, 3rd ed: American College of Chest Physicians Evidence-Based Clinical Practice Guidelines. Chest 2013;143:e314S-40S. 
44. Maconachie R, Mercer T, Navani N, et al. Lung cancer: diagnosis and management: summary of updated NICE guidance. BMJ 2019;364:11049.

45. Robinson A, Vella ET, Ellis PM, et al. Recommendations for the treatment of patients with clinical stage III nonsmall cell lung cancer: endorsement of the 2019 National Institute for Health and Care Excellence Guidance and the 2018 Society for Immunotherapy of Cancer Guidance. 2020 .

doi: $10.21037 /$ ccts-20-101

Cite this article as: Iseli T, Süveg K, Fischer GF, Glatzer M, Putora PM. Management of stage IIIA NSCLC: the role of radiotherapy-a narrative review. Curr Chall Thorac Surg 2022;4:14.
46. Evison M, McDonald F, Batchelor T. What is the role of surgery in potentially resectable N2 non-small cell lung cancer? Thorax 2018;73:1105.

47. Glatzer M, Panje CM, Sirén C, et al. Decision making criteria in oncology. Oncology 2020;98:370-8.

48. Iseli T, Berghmans T, Glatzer M, et al. Adverse events reporting in stage III NSCLC trials investigating surgery and radiotherapy. ERJ Open Res 2020;6:00010-2020. 